\title{
Fractal Hilbert Sensor to Detect Partial Discharge on Transformer
}

\author{
H H Sinaga $^{1 *}$, H B H Sitorus ${ }^{1}$, D Permata ${ }^{1}$, and N Soedjarwanto ${ }^{1}$ \\ ${ }^{1}$ Department of Electrical Engineering, Faculty of Engineering, Universitas Lampung, Jalan Prof. Soemantri Brojonegoro \\ No. 1, Bandar Lampung, 35143 Indonesia \\ *Email: herman.h.sinaga@eng.unila.ac.id
}

Article Information
Received:
10 October 2019
Received in revised form:
20 November 2019
Accepted:
15 December 2019
Volume 1 , Issue 2 , December 2019
pp. $94-100$
CUniversitas Lampung
http://dx.doi.org/10.23960/jesr.v1i2. 31

\begin{abstract}
The design of fractal Hilbert sensor is presented in this paper. The sensor is intended to detect partial discharge (PD) in transformer insulation. The fractal Hilbert sensor designed using 4 order fractal and etching on single layer PCB board. The Hilbert fractal type is chosen as this type of sensor can be built on smaller area compare to other types. The sensor overall dimension is limited to $5 \times 5 \mathrm{~cm}$ as it is proposed to be use to detect the PD presence in transformer. The sensor sensitivity is tested using a closed type TEM cell. After the sensitivity of the sensor is tested the sensor then applied to detect the PD signals emitted by void PD defect model. The results show the sensor has sensitivity as high as $10 \mathrm{~dB}$. The sensor also has capability to detect the PD signals generated by the PD defect source. The waveforms captured by the sensor show the sensors can capture high frequency pulse generated by the PD source.
\end{abstract}

Keywords: partial discharge, Fractal Hilbert Sensor, sensor sensitivity.

\section{INTRODUCTION}

$\mathrm{T}$ ransformer insulation system designed to be able to withstand all pressure, such as electric stress and mechanical during its designed time utilizing period. However sometimes, due to the insulation imperfection, such as defects might occur inside the insulation of the operated transformer although rigorous inspection already carried out during testing period before installing the transformer. After certain period of times at the imperfection spot, Partial Discharge (PD) can occurs due to the high electric stress which is above the inception PD voltage. If let grow over time, the PD can cause insulation deterioration which might lead to catastrophic failure of the transformer. Thus, the PD can be used as a prediction tool to examine the insulation deterioration.

The PD event can be detected by the product resulted by the PD events, such as discharge current, sound, gas (due to chemical reaction), heat, light and electromagnetic signals. Partial discharge detection method by capture the electromagnetic signals is one of developed lately to detect Partial Discharge at transformer insulations [1]. Using appropriate sensor, the electromagnetic signals generated by the PD source can be detected and capture then recorded by using digitizer or oscilloscope.

\section{A. PD detection on Insulation of the electrical equipment}

The presence of defect such as cavity or metal particle trapped inside insulation can cause high electric stress local. Such high local tress can cause local breakdown which is known as partial discharge. When discharge occurs, electric field will accelerate and decelerate electron which is in stationary condition beforehand. During the accelerate and decelerate process, electron produce time varying electric and magnetic field. The combination of the time varying electric and magnetic field than produce electromagnetic wave which radiated to any direction from the PD event location.

The UHF PD detection method is known to have advantages over conventional PD detection methods. This is mainly due to the higher immunity of the UHF method to noise. As described before, the UHF method works in a frequency range of $300 \mathrm{MHz}$ to $3000 \mathrm{MHz}$ while the noise in a substation is primarily below a few 
$\mathrm{MHz}$ [2]. Thus, the UHF range is relatively noise-free, except for interferences from well-known communication sources such as digital TV/Radio and mobile phone.

The UHF detection method has been widely used to detect the occurrence of PD sources in high voltage equipment, especially in Gas Insulated Switchgear (GIS). More recently, the UHF detection method has also been applied to detect PDs in power transformers $[3,4]$.

\section{B. UHF PD detection in transformer}

The transformer is one of the most important equipment items in electrical power system networks. Any failures that lead to transformer outage must be avoided. A correlation between the long-term partial discharge activity and the insulation failure has not been proven yet. However, it is believed that transformer failure is mostly due to failure in the insulation and this is usually started by the occurrence of partial discharges $[5,6]$. Thus, it is important to assess the transformer insulation to establish the condition of the transformer.

The presence of defects such as a cavity or a metal particle trapped in the insulation is undesirable. These defects will enhance the local electric stress and if it is excessive, local breakdown will occur which is known as partial discharge. During the discharge, the electric field will rapidly accelerate and decelerate electrons which are initially at rest. As a result of the timevarying electric and magnetic fields, electromagnetic waves are produced and radiated outward from the PD source.

UHF PD measurement has been shown to be effective in detecting PDs in GIS and is now increasingly being applied to monitoring the condition of transformer insulation [7,8]. One may wonder if the UHF measurement in GIS can be adopted and applied to power transformers. In GIS, PD pulse rise times can be as short as 50 ps [9]; but in power transformers, the fastest rise time is around $0.9 \mathrm{~ns}$ due to permittivity of oil higher than SF6 gas [10]. Nevertheless, pulses in the UHF range (300 -3000 MHz) will be excited for such a rise time [11]. Thus, UHF measurement can be adopted to PD measuring in transformers.

\section{SENSORS TO DETECT PD IN TRANSFORMER}

The purpose of transformer monitoring extends from PD detection and recognition to PD localization. To be able to undertake UHF PD transformer monitoring, a sensor with capabilities to detect signals in the UHF frequency range is needed. For power transformer monitoring, the UHF sensor is inserted into the transformer tank to capture the electromagnetic waves emitted by the PD source. There are two ways of installing the sensor: via the oil drain valve [12] or the dielectric window [8]. The size of the oil drain valve imposes a constraint on the sensor dimension, while the dielectric window can be created with an appropriate size to accommodate the sensor. However, the placement of a dielectric window sensor needs an additional hole to be fabricated on the transformer tank. As for the oil valve sensor, this is not required because the sensor can be easily retrofitted into the transformer via the existing built-in oil drain valve [12].

Typically, the sensor is a monopole type. It can be inserted via the oil drain valve. The size of the sensor is usually limited by the diameter and length of the oil drain. A typical oil drain valve sensor size is $5 \mathrm{~cm}$ in diameter, and $10 \mathrm{~cm}$ in length [12]. The shape of the sensor can be a short monopole [12,13], plate, zigzag or conical $[12,14]$ or any shape as long as it is able to be fitted to the drain valve. The sensitivity of this kind of sensor is affected by the depth of the sensor insertion [12]. The deeper the sensor is inserted, the higher the magnitude of the PD signals acquired. However, the sensor must not initiate breakdown due to the high electric stress at the tip of the sensor [8]. To reduce electric stress, the sensor can be encapsulated in some dielectric material [8].

For a dielectric window, the sensors usually have a planar shape [8]. The sensor can be a micro-strip sensor [15-18], log-spiral, spiral [8,19] or fractal [20-22]. This kind of sensor is usually etched on the surface of a dielectric material, using the same process as in making electronic printed circuit boards (PCB). The sensor is etched on the PCB with dimensions proportional to the working frequency of the sensor. In 16, the miniaturization of the microstrip UHF antenna was discussed by applying an impedance matching technique. This technique can reduce the antenna dimensions to $5 \times 5 \mathrm{~cm}$ for example. However, even though the antenna works in UHF range, the bandwidth is very limited. To increase the bandwidth, [17] designed a microstrip antenna using sandwich substrate. As a result, the frequency range of the antenna has a very wide range from $30 \mathrm{MHz}$ to 3000 MHz. However, to manufacture this microstrip antenna is not practical.

Most of the measuring arrangements are unbalanced systems, i.e. the input to the measuring system is a coaxial cable which consists of a live input and ground. While planar sensors are usually crafted as a balanced system, an unbalanced system is also possible, such as a circular or single arm log-spiral or spiral. Thus to connect the sensor to the measuring system, a converter from balanced to unbalanced is needed. This connector forms part of the sensor and is called a balun.

The antennae and measurement system usually have different impedances. The typical value for measuring 
equipment is $50 \mathrm{ohms}$ and sometimes also $75 \mathrm{ohms}$ while the sensor impedance varies from a hundred to a few hundred ohms [20]. The balun, besides acting as a converter from balanced to unbalanced, is also used to provide impedance transformation between different impedance values. The balun design is based on the working frequency and the impedance difference [20,23]. Duncan [23] created a balun with a high frequency bandwidth which increased up to 100:1 using the tapered method. The balun is created by using a tapered coaxial with a specific diameter. The impedance transition is achieved by cutting open the outer wall of the coax so that a cross-section view shows a sector of the outer conductor removed [23]. This balun has a simple design and can use the regular coaxial cable. Also, the impedance transition is very smooth as a result of the tapered opening. However, the length of this balun is too long for practical application. Using a standard coaxial cable, for transition of 120 ohms to 50 ohms over UHF frequency range (300 $3000 \mathrm{MHz}$ ), the balun can be more than $50 \mathrm{~cm}$ in length [24,25] which is impractical to apply in power transformers.

To decrease the balun length, a material with higher permittivity $[25,26]$ can be used. Using special high permittivity material, the balun length can be reduced to less than $10 \mathrm{~cm} \mathrm{[26]} \mathrm{which} \mathrm{is} \mathrm{feasible} \mathrm{in} \mathrm{enabling}$ connection to the sensor. However, it is more difficult to fabricate a balun using such a material.

To date, advances in printed circuit board (PCB) and microwave integrated circuit (MIC) substrate have meant that the micro-strip balun design has become more popular. The balun can be created by etching the PCB surface to form a specific pattern. In [20] the micro-strip balun design is set with a frequency range of $0.1 \mathrm{GHz}$ to $3.85 \mathrm{GHz}$. The overall length of the balun is only $46 \mathrm{~mm}$ by using a standard single layer PCB. As the impedance transitions use several stages, the transition is not as smooth as the tapered balun. However, this balun is much easier to fabricate, uses cheaper material [20] and is sufficient to interface with the antenna and the measuring equipment.

In this paper discussed the design of fractal Hilbert sensor due to advantage of this type of sensor such as much smaller dimension [21] and also has higher bandwidth [22]. The sensor also can be easily fabricated on a single board PCB. As the fractal Hilbert sensor is a monopole type antenna, there is no need a converter when connected to a coaxial cable, which is needed for dipole antenna type.

The placement of the UHF sensors to detect PD events in transformers is usually at fixed locations. The locations of sensor installation depend on the type of sensor and transformer construction, as described in a previous section. As the PD can occur in any location in the transformer, the electromagnetic signal path from the PD source to the sensor can be affected by the structure inside the transformer. The signal path is also affected by the density of the insulation oil in the transformer tank which can also be subjected to variations in temperature (caused by loading changes). In addition, the live active parts of the transformer can obstruct electromagnetic signals and cause signal attenuation whereby the signal becomes not linear to the distance. This will cause conversion of the signal magnitude detected by the UHF sensor to an equivalent pC level to become difficult [27].

Other also recommend that sensitivity verification for UHF method as a substitution for calibration. Sensitivity verification can be used on-site to determine the minimum sensitivity of the measuring system [28]. Although this recommendation is only for GIS, with some adjustment this method can also be applied to power transformers which use oil insulation. The sensitivity of the UHF measuring method is very dependent on the type of sensor, on the type of the PD defect, and on the location of the PD source $[29,30]$.

\section{Sensor Fractal Hilbert to Detect PARTIAL DischaRge}

Fractal Hilbert sensor can be constructed with smaller dimension compare to different type of sensor. This is mainly due to the nature of fractal Hilbert with its construction so the total length of the conductor is far longer with the similar area size. Figure 1 shows Fractal Hilbert pattern with similar area size but different conductors' length. The length and wide of the sensor area can be easily adjusted to the requirement application. In this paper discussed the fractal Hilbert sensor with length and wide of the sensor as $5 \mathrm{~cm}$. Dis dimension meet the criterion of the oil drain valve of a power transformer [21,31].
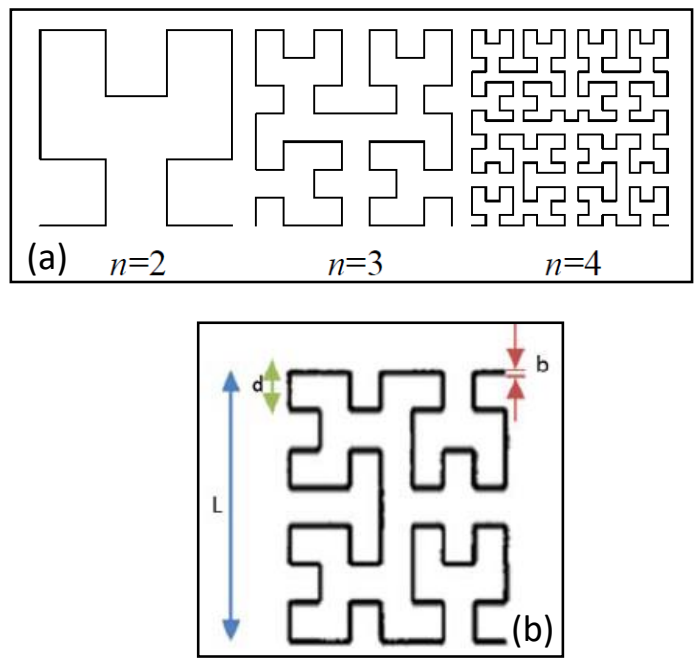

Figure 1. Fractal Hilbert pattern (a) similar size but different order and (b) Fractal Hilbert dimension 
Sensor Hilbert parameters can be seen from Figure 1.b. Fractal Hilbert sensor has a nearly symmetrical pattern with distance of every conductor strips are similar. After the length/wide of the sensor area are selected than the conductor segments distance can be calculated as:

$$
d=\frac{L}{2^{n}-1}
$$

Where, $\mathrm{L}$ as the length/wide of the sensor, $\mathrm{d}$ is the conductor segments distance, and $\mathrm{n}$ is the Hilbert order. The higher $\mathrm{s}$ the $\mathrm{n}$ value the segment number is higher too.

The sensor Hilbert order determined from the desired resonance frequency value. Usually for Hilbert sensor, resonance frequency must be higher than $300 \mathrm{MHz}$ [32]. The resonance frequency can be calculated using equation below:

$$
\begin{array}{r}
\frac{\mu_{0} k \lambda}{4 \pi}\left[\log \left(\frac{8 k \lambda}{4 b}\right)-1\right]=\frac{\mu_{0}}{\pi} s\left[\log \left(\frac{8 s}{b}\right)-1\right]+ \\
m \frac{\eta}{\pi \omega} \log \left(\frac{2 d}{b}\right) \tan \beta d
\end{array}
$$

Where, $\mu_{0}$ is the air permeability, $\lambda$ is the wavelength, $\eta$ is the intrinsic impedance, $\beta$ is phase constant and $\mathrm{k}$ is an odd number which define the kth resonance frequency. The segment number $\mathrm{m}$ and segment total length $\mathrm{s}$ can be determined by:

$$
\begin{gathered}
m=4^{n-1} \\
s=\left(2^{2 n-1}-1\right) d
\end{gathered}
$$

Conductor width $\mathrm{b}$ can be calculated using the matching impedance equation:

$$
Z_{0}=\frac{\eta}{\pi} \log \left(\frac{2 d}{b}\right)
$$

The value of the matching impedance usually taken as $50 \mathrm{ohms}$, the impedance value of most laboratory equipment. Though impedance value of 75 and 100 ohm also can be used. However, if the impedance value is different than the measurements equipment than a balun is needed to match and bridge the different impedance.

The Hilbert fractal sensor has square dimension of $5 \times 5 \mathrm{~cm}$. The sensor designed to work at the frequency range of 300 to $500 \mathrm{Mhz}$. This frequency range fall is chosen as the purpose of the sensor is to detect PD in oil insulated transformer. Full UHF frequency range $(300 \mathrm{MHz}-3000 \mathrm{MHz}$ ) as usually use in GIS PD detection, might not needed as the PD signal in oil insulation has much lower than in GIS [33].

By applied equation 1 to 5 , segment number and segment width of the fractal pattern are calculated. Then the pattern printed and etching on a single layer PCB board. The etching sensor pattern on a PCB board is shown in Figure 2.

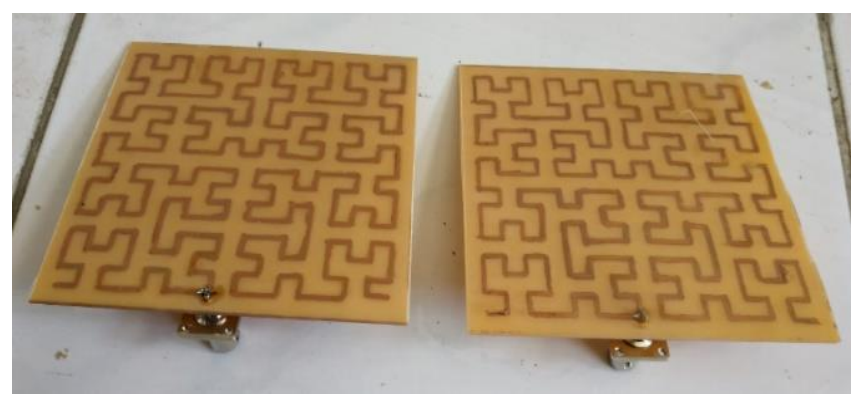

Figure 2. Fractal Hilbert etching on a PCB board

The calculated results of the sensor dimension can be seen in table 1. The Hilbert order is 4 , to limit the complexity of the sensor pattern thus to make sensor fabrication easier.

Table 1. Calculated sensor Fractal Hilbert Dimension

\begin{tabular}{|l|l|}
\hline Sensor dimension & $5 \times 5 \mathrm{~cm}$ \\
\hline Conductor width & 0.3 \\
\hline Segment number & 15 \\
\hline Impedance & $50 \mathrm{Ohm}$ \\
\hline Order number & 4 \\
\hline
\end{tabular}

\section{RESULTS AND DISCUSSION}

\section{A. Sensor Sensitivity Test}

Sensor sensitivity test conduct by using TEM -cell with experiment arrangement is shown in Fig 3. The sensor placed inside the TEM cell connected to an oscilloscope. The input signals generated by pulse generator. The pulse generator also connected to the oscilloscope. The pulse generator is set to produce a step-pulse to imitate the high frequency generated by the PD pulse.

The sensor showed high magnitude with oscillation up to almost $50 \mathrm{~ns}$, which was caused by the length and structure of the fractal Hilbert being lengthy and complex. The typical sensor attenuation is around 10 $\mathrm{dB}$.

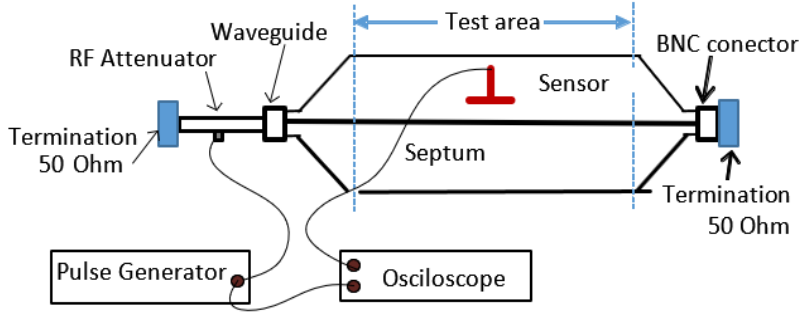

Figure 3. Fractal Hilbert sensor sensitivity test 


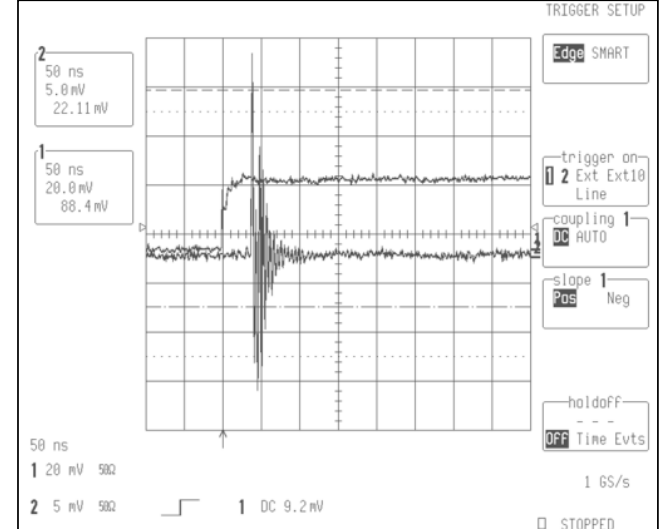

Figure 4. Typical Sensor response to step-pulse

\section{B. Sensor to Detect PD signals}

The experimental setup is shown in Figure 5. The PD source and the sensor installed inside aluminum box. High voltage connection to a discharge source placed inside the tank was made via a high-voltage bushing. The fractal Hilbert sensor was placed inside the aluminum box and connected to an oscilloscope outside.

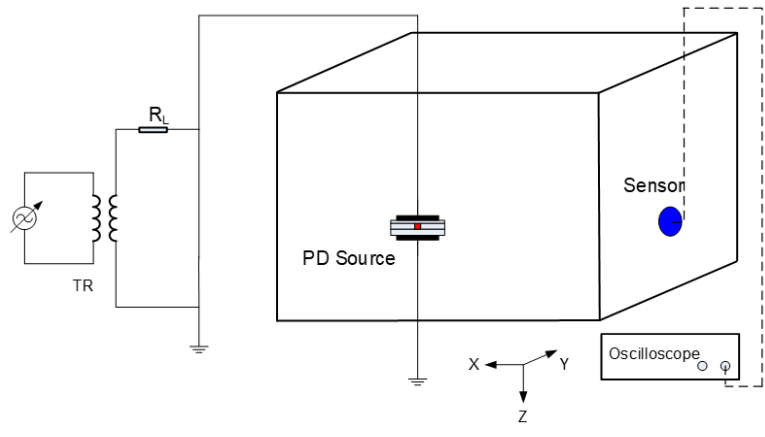

Figure 5. Experiment diagram of the sensor ability to capture PD signals

Standard transformer pressboard was used to construct artificial PD defects. Cavity discharge was simulated using two flat electrodes separated by a stack of three layers of pressboard with a $2 \mathrm{~mm}$ diameter hole in the middle layer.

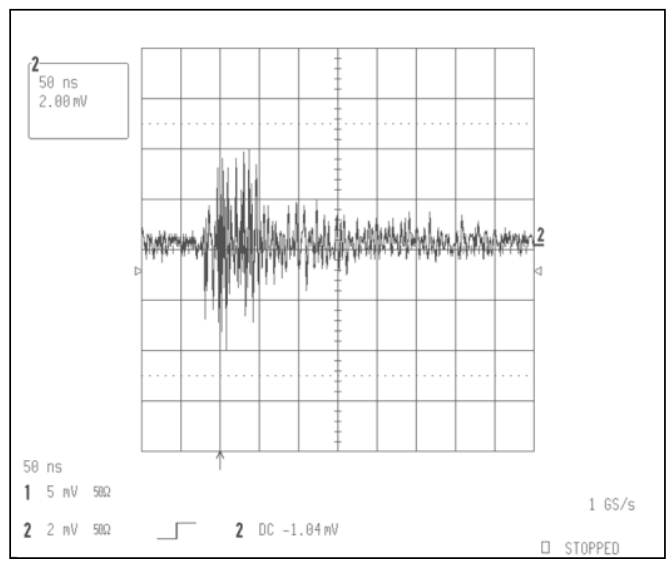

(a)

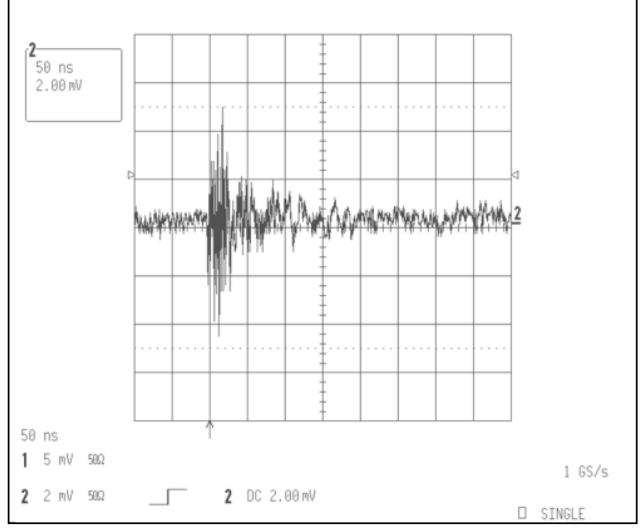

(b)

Figure 6. Typical of the recorded PD waveform ( $a$ and $b$ ), the PD signals captured by the fractal Hilbert sensor

The PD signals capture by the Fractal Hilbert sensor are shown in Fig 6. The sensor shows capability to detect the PD signal and recorded using oscilloscope. The PD waveform produce by the PD defect model has a very high frequency as shown by the waveform with very high oscillation on short duration. Figure 6.a shows the PD has wider pulse thus longer time response, whilst PD pulse of the Fig 6.b has narrower pulse thus shorter time response. Nevertheless, the figure 6 shows that the designed fractal Hilbert sensor capable to detect the PD signal generated by PD source. Thus, these results justify the ability of the fractal Hilbert sensor to detect the PD signal.

\section{CONCLUSIONS}

In this paper designed fractal Hilbert sensor with dimension of $5 \times 5 \mathrm{~cm}$. The sensor constructed using a single layer PCB. The designed fractal Hilbert sensor has high sensitivity with attenuation as much as $10 \mathrm{~dB}$. The sensor also capable to capture the PD signals generated by void PD defect source.

\section{ACKNOWLEDGMENT}

The authors would like to express their gratitude to Kemenristek-DIKTI Indonesia for supporting this research. This research was supported by KemenristekDIKTI Indonesia with the Fundamental Research Grant, contract number: 857/UN26.21/PN/2019.

\section{REFERENCES}

[1] J. Fabian, M. Neuwersch, C. Sumereder, M. Muhr and R. SchwarZ. (2014). State of the Art and Future Trends of Unconventional PD-Measurement at Power Transformers. Journal of Energy and Power Engineering, vol. 8, pp. 1093-1098. 
[2] IEC Standard Evaluation and qualification of electrical insulation systems, IEC Standard 60505, 2004.

[3] M. D. Judd, L. Yang and I. B. B. Hunter. (2005). Partial Discharge Monitoring for Power Transformers Using UHF Sensors Part 1: Sensors and Signal Interpretation. IEEE Electrical Insulation Magazine, vol. 21 (2), pp. 514.

[4] M. D. Judd, G. P. Cleary, C. J. Bennoch, J. S. Pearson and Breckenridge. (2002). Power transformer monitoring using UHF sensors: site trials Electrical Insulation. Proceeding of The IEEE International Symposium on Electrical Insulation, pp. 145-149.

[5] T, J. Gallagher and A. J. Pearmain, "High Voltage: Measurement, Testing and Design". New York, USA: John Wiley \& Sons, 1983.

[6] G. C. Stone. (2005). Partial Discharge Diagnostics and Electrical Equipment Insulation Condition Assessment" IEEE Trans. on Dielectrics and Electrical Insulation. Vol. 12 (5), pp. 891-903.

[7] M. D. Judd, L. Yang and I. B. B. Hunter. (2005). Partial discharge monitoring for power transformers using UHF sensors Part 2: Field Experience. IEEE Electrical Insulation Magazine. Vol. 21 (3). pp. 5-13.

[8] D. Warne and A. Haddad, "Advance in High voltage Engineering", London, UK: Institution of Electrical Engineers, 2004.

[9] M. D. Judd and O. Farish. (1998). High Bandwith Measurement of Partial Discharge Current Pulses. Proceedings of International Symposium on Electrical Insulation, pp. 436-439.

[10] G. P. Cleary and M. D. Judd. (2006). UHF and current pulse measurements of partial discharge activity in mineral oil. IEEE Proc. of Science Measurement Technology. Vol. 153 (2), pp. 47 - 54.

[11] M. D. Judd, O. Farish and B. F. Hampton. (1996). The excitation of UHF signals by partial discharges in GIS. IEEE Trans. on Dielectrics and Electrical Insulation. Vol. 3 (2), pp. $213-228$.

[12] J. L. Roldan, T. Tang and M. Gaskin. (2008). Optimisation of a Sensor for Onsite Detection of Partial Discharges in Power Transformers by the UHF Method. IEEE Trans. on Dielectrics and Electrical Insulation. Vol. 15 (6), pp. 1634-1639.

[13]T. Pinpart and M. D. Judd. (2009). Experimental comparison of UHF sensor types for PD location applications. Proceedings of Electrical Insulation Conference (EIC 2009), pp. 26- 30.

[14]P. Agoris, S. Meijer and J. J. Smith. (2007). Sensitivity Check of an Internal VHF/UHF Sensor for Transformer Partial Discharge Measurements. Proceeding of The Powertech '07 Conference, pp. 2065 - 2069.

[15] A. Ando, K. Kagoshima, A. Kondo and S. Kubota. (2008). Novel Microstrip Antenna with Rotatable Patch Fed by Coaxial Line for Personal Handy-Phone System Units. IEEE Trans. On Antennas and Propagation. Vol. 56 (8), pp. 2747-2751.

[16] G. Marrocco. (2008). The Art of UHF RFID Antenna Design: Impedance-Matching and Size-Reduction Techniques. IEEE Antennas and Propagation Magazine. Vol. 50 (1), pp. 66-79.

[17] A. Erentok and R. W. Ziolkowski. (2008). MetamaterialInspired Efficient Electrically Small Antennas. IEEE
Trans. On Antennas and Propagation. Vol. 56 (3), pp. 691-707, 2008.

[18] T. Ju, X. Zhongrong, Z. Xiaoxing and S. Caixin. (2007). GIS partial discharge quantitative measurements using UHF microstrip antenna sensors. Proceeding of Conf. on Electrical Insulation and Dielectric Phenomena (CEIDP 2007. pp. 116- 119.

[19] A. Roy, Ghosh and A. Chakrabarty. (2007). Wideband Performance of Dielectric Loaded Monopole Transreceive Antenna System. Proceeding of Intl. Conf. on Industrial and Information Systems (ICIIS 2007). pp. 181-185.

[20] J. Thaysen, K. B. Jakobsen and J. Appel-Hansen. (2000). A Wideband Balun-How Does it Work. Applied Microwave and Wireless. Vol. 12 (10), pp. 40-50.

[21] W. Yongqiang, W. Zhuang and L. Jianfang. (2017). UHF Moore Fractal Antennas for Online GIS PD Detection. IEEE Antennas and Wireless Propagation Letters. Vol. 16, pp. $852-855$.

[22] J. Li, T. Jiang, C. Cheng and C. Wang. (2013). Hilbert fractal antenna for UHF detection of partial discharges in transformers. IEEE Trans. on Dielectrics and Electrical Insulation. Vol. 20 (6), pp. 2017 - 2025.

[23] J. W. Duncan and P. V. Minerva. (1960). 100:1 Bandwidth Balun Transformer. Proceedings of the Institute of Radio Engineers (IRE). Vol. 48 (2), pp. 156164.

24] H. H. Sinaga, B. T. Phung and T. R. Blackburn. (2009). Design of ultrahigh frequency sensors for detection of partial discharges. Proceeding of International Symposium on High Voltage Engineering, Cape Canaveral, South Africa - 2009.

[25] M. D. Judd, O. Farich and B. F. Hampton. (1995). Broadband coupler for UHF detection of partial discharge in gas insulated substations. IEEE Proceedings on Science, Measurement and Technology. Vol. 142 (3), pp. $237-243$.

[26] J. G. Choi, S. W. Yi and K. H. Kim. (2004). Development of a novel tapered balun for the UWB UHF coupler. Conference proceedings of $26^{\text {th }}$ International Power Modulator Symposium. Pp, 493 - 496.

[27] S. Coenen, S. Tenbohlen, S. M. Markalous and T. Strehl. (2008). Sensitivity of UHF PD measurements in power transformers. IEEE Trans. on Dielectrics and Electrical Insulation. Vol. 15 (6), pp. 1153-1158.

[28] T. F. Cigre. (1999). PD Detection System for GIS: Sensitivity Verification for the UHF Method and the Acoustic Method. Electra. Vol. 183, pp. 75-87.

[29] S. Coenen, S. Tenbohlen, S. M. Markalous, and T. Strehl. (2009). Sensitivity limits of UHF PD measurements on power transformers. Proceedings of International Symposium on High Voltage Engineering, Cape Caneveral - South Africa.

[30] A. Troeger, U. Riechert, S. Burow and S. Tenbohlen. (2010). Sensitivity Evaluation of Different Types of PD Sensors for UHF-PD-Measurements. Proceeding of International Conference on Condition Monitoring and Diagnostics, pp. 839-842.

[31] J. L. Roldan, T. Tang and M. Gaskin. (2008). Optimisation of a Sensor for Onsite Detection of Partial Discharges in Power Transformers by the UHF Method. IEEE Trans. on Dielectrics and Electrical Insulation. Vol. 15 (6), pp.1634 - 1639. 
[32] A. A. Zahed, A. H. El-Hag, N. Qaddoumi, R. Hussein and K B Shaban. (2017). Comparison of different fourth order Hilbert fractal antennas for partial discharge measurement. IEEE Trans. on Dielectrics and Electrical Insulation. Vol. 24 (1), pp. $175-182$.
[33] G. P. Cleary and M. D. Judd (2006). UHF and current pulse measurements of partial discharge activity in mineral oil". IEE Proc. of Science Measurement Technology. Vol. 153 (2), pp. $47-54$. 